\title{
Algunos datos clínicos ayudan a seleccionar qué pacientes con adenopatías periféricas requieren biopsia
}

Application of a Prediction Rule to Select which Patients Presenting with Lymphadenopathy Should Undergo a Lymph Node Biopsy. Vassilakopoulus T, Pangalis G. Medicine 2000;79:338-47.

\section{Objetivo}

Desarrollar una regla de predicción clínica basada en datos del interrogatorio y examen físico para discriminar qué pacientes con linfadenopatias periféricas se benefician con una biopsia de ganglio linfático.

\section{Diseño}

Desarrollo y validación de una regla de predicción clínica utilizando datos de una misma población.

\section{Lugar}

Servicio de hematologia de la universidad de Atenas, Grecia.

\section{Pacientes}

Se incluyeron 475 pacientes $\geq$ de 14 años con adenopatías periféricas vistos en forma ambulatoria. Se excluyeron pacientes con antecedentes de biopsia o aspiración de ganglio, perfil leucémico o diagnóstico previo de VIH. La mediana de seguimiento fue de 69 meses (rango: 45-108).

Descripción del test y del test de referencia (gold standard) Se desarrolló la regla con los 315 pacientes iniciales, y se validó su funcionamiento con los 160 finales.

Uno de los autores examinó a los pacientes en cuanto a las características de los ganglios, hígado, bazo y si había fiebre, perdida de peso, sudores nocturnos, odinfagia, rash y prurito generalizado. La decisión final en cuanto a la necesidad de biopsia era tomada por el otro autor quien también examinó a los pacientes pero no registraba con detalle los datos. Finalmente se analizó retrospectivamente si la biopsia ayudo a un diagnóstico y tratamiento especifico. Los pacientes en los que no realizó biopsia era porque el diagnóstico etiológico se estableció con otros métodos, o por ser linfadenopatías inespecíficas que no evolucionaron durante el seguimiento. 83 pacientes tuvieron enfermedades que requirieron biopsia, mientras 232 se diagnosticaron sin recurrir a la misma.

\section{Medición de Resultados Principales}

Características operativas del test (sensibilidad, especificidad, valor predictivo positivo y negativo*).
Regla de Predicción Clínica y sugerencias de uso

Paso 1: Determinar puntaje

\begin{tabular}{l|c}
\hline Variable & Puntos \\
\hline Edad $: \geq 40$ & 5 \\
\hline Dolor $: \mathrm{Si}$ & -5 \\
\hline Tamañot: $<1 \mathrm{~cm} 2$ & 0 \\
\hline \multicolumn{1}{c|}{$1-3,99 \mathrm{~cm} 2$} & 4 \\
\hline \multicolumn{1}{c|}{$4-8,99 \mathrm{~cm} 2$} & 8 \\
\hline$>9 \mathrm{~cm} 2$ & 12 \\
\hline Prurito generalizado & 4 \\
\hline Adenopatía supraclavicular & 3 \\
\hline Textura durał & 2 \\
\hline Consțante & -6 \\
\hline
\end{tabular}

*: Al menos 1 ganglio doloroso

†: Se multiplican las 2 dimensiones (ej: un ganglio de $2 \times 2$ tiene $4 \mathrm{~cm} 2$ )

‡: Al menos 1 ganglio de textura dura.

Por ejemplo, una paciente de 55 años (5 puntos) con un ganglio cervical de $3 \mathrm{~cm} 2$ (4 puntos) doloroso ( -5 puntos) y duroelástico tendría un puntaje de $5+4-5-6$ (constante) $=-2$, por lo que no requeriría biopsia para el diagnóstico.

\section{Paso 2: Sumar el puntaje}

Si el mismo es $\geq 1$ : Biopsia

Se debe realizar inicialmente una historia y examen físico con énfasis en las variables de la regla de predicción. Aunque el VIH fue raro en esta serie, pensarlo si hay factores de riesgo. Luego se debe calcular el puntaje. Si este es $>=1$ la probabilidad de tener una enfermedad que requiera la biopsia es alta. Antes de la biopsia descartar infección por Toxoplasma, Epstein Barr. Si el puntaje es 0 o menor, hacer el laboratorio apropiado, y si con este no se logra el diagnóstico se debe seguir al paciente de cerca con un diagnóstico operativo de linfadenopatía inespecífica. El seguimiento periódico es esencial en estos pacientes sin indicación convincente de biopsia.

\section{Conclusiones}

Esta regla de predicción clínica sirve para detectar aquellos pacientes con adenopatías periféricas que se beneficiaran con la realización de una biopsia.

\section{Resultados Principales}

\begin{tabular}{|c|c|c|c|c|}
\hline & $\begin{array}{l}\text { Sensibilidad } \\
\text { (IC95\%) }\end{array}$ & $\begin{array}{l}\text { Especificidad } \\
\text { (IC95\%) }\end{array}$ & VPP & VPN \\
\hline rupo Deris & $95,2(88,1-98,1)$ & $81(75,4-85,6)$ & 64,2 & 97,9 \\
\hline Grupo Validación $(n=160)$ & $96,9(83,9-99,5)$ & $91,4(85,1-95,2)$ & 73,8 & 99,2 \\
\hline Global & $95,7(90,1-98,2)$ & $84,7(80,6-88,1)$ & 66,7 & 98,4 \\
\hline
\end{tabular}

\section{Comentario}

Existe poca evidencia acerca de cuando biopsiar una adenopatía periférica. Se han publicado 2 trabajos retrospectivos que analizan esta decisión, pero la edad de los pacientes ( 9 a 25 años) y el ser una población altamente seleccionada (biopsiados por algún motivo) limitan su aplicación general.1-2

El presente trabajo muestra varios puntos interesantes: Los predictores fueron recolectados prospectivamente, la regla es fácil de usar, tiene validez de contenido, y sugiere un curso de acción mas que predecir un evento futuro. Si bien lo ideal es que la realización de la biopsia se hubiese hecho sin el conocimiento de los predictores, aquí no resulta tan importante debido a que la biopsia no esta tan sujeta a interpretación, además resulta imposible hacerla sin revisar primero al paciente, por otra parte los patólogos si estaban ciegos con respecto a las características de los pacientes.

Tampoco se describe en el estudio la variabilidad interobservador, lo cual es necesario para 2 variables como la textura y el tamaño que son interpretadas en forma variable por distintos observadores.3-4

En síntesis, representa un buen primer paso para su uso clínico, sobretodo para llamar la atención de variables clínicamente importantes en una adenopatía. Es importante que antes de recomendarla en nuestra población sea validada en atención primaria (como ejemplo, la prevalencia de cáncer en este estudio, hecho en un servicio de hematología, fue del $18 \%$ cuando en atención primaria es del 1\%), y analizar si tiene aceptación practica por parte de los médicos.

\section{Dr. Cristian Dellepiane [ Unidad de Medicina Familiar y Preventiva. Hospital Italiano de Buenos Aires ]}

Referencias

1.Slap G, Brooks J, Schwartz S. When to perform Biopsies of Enlarged Peripheral Lymph Nodes in young patients. JAMA 1984; 252: 1321-6.

2. Slap G, Connor J, Wigton R. Validation of a model to identify young patients for Lymph Node Biopsy. JAMA 1986; 255: 2768-73.

3. Laupacis A, Sekar N, Stiell I. Clinical Prediction Rules: A review and suggested modifications of methodological standars. JAMA 1997; 277: 488-94.

4. McGinn T, Guyatt G, Wyer P. User's Guides to the Medical Literature: How to use articles about Clinical Decision Rules. JAMA 2000; 284: 79-84. 